\title{
Comparative Analysis of the Online Recruiting Platforms using Utility-related Factors
}

\author{
Gabriel CALIN, Iulia BIVOLARU, Paula IVAN \\ Bucharest University of Economic Studies, Romania \\ calingabriel89@gmail.com, iulia.bivolaru24@gmail.com, paulaivan92@gmail.com
}

\begin{abstract}
The purpose of this study was to investigate the possibility of defining factors which can be used as a measure of the utility of different types of online recruiting platforms and then measure these factors for different such platforms. Three platforms were chosen: freelancer.com, stagiipebune.ro and hipo.ro. We measured 25 features, which together gave a value for the utility of each platform. Based on these values, it is clear that important features which make a difference nowadays are integration with social media and mobile applications and also the way the information is presented. Information should be easy to follow, but not too scarce.
\end{abstract}

Keywords: E-Recruiting, Utility Factors, Comparative Analysis, Features

1 Introduction

Online recruiting is a revolutionary way of finding the right people to do the right job. It allows for a complete reorganization of the HR systems, one in which the central part of the recruitment process is not having a lot of applicants coming to your premises, but one which filters the potential candidates beforehand, based on different criteria.

A very important aspect is the financial one. Having people use an online platform will make a lot of cash savings in the HR department. In terms of recruiting, such a platform may allow candidates to create their own career pages, while the companies can have their own pages. This translates in a greater transparency for both the candidate and the company. The candidate can visit the company's page and see all the available jobs, along with a detailed description, required or nice to have skills. The company can visit the candidate's page and see his resume before deciding whether he might be fit for the job or not.

But maybe the most important advantage of online platforms compared to the classical way of hiring new people is the rapidity. Forgotten are the days when a HR employee had to go through dozens, maybe hundreds of papers in order to decide on a more limited number of people which were then asked to come for an interview. Now, everything resumes to applying a filter through all the potential candidates and the platform will only show the relevant ones. Also, the applicant doesn't have to send the resume physically to every company which has a position that the candidate is interested in. All he has to do is fill the resume once (online) and then, usually, the process of applying to a job resumes to pressing a button on the screen.

One of the central parts of having a successful recruitment platform is to convince people to use your platform. As the developer of such a platform, a lot of questions can be asked about the future implementation of the platform. What features are the most demanded in today's online environment? Which of them should be implemented with priority? Which should be the target group of the platform? Maybe students or freelancers? Or maybe a more general platform, suitable for everyone is better? As the user of the platform, other questions come up. What platform should I decide to use in order to increase my chances of getting the right job? What features do different platform have and which of them would help me the most?

Starting with the next section, we will analyze the different ways in which different recruitment platforms are doing it. We will take successful platforms and see what makes them different than the rest. In the end we'll draw conclusions about the functionalities which seem to attract the most users to these platforms.

This kind of review comes as a necessity as there is no clear way right now about how different platforms differ from one another. It

DOI: 10.12948/issn14531305/20.2.2016.02 
would be very useful, both for the users but also for the developers of such platforms, to have a structured overview about different online platforms. Based on the conclusions we will draw, a user might decide about the features to look for in online recruitment platforms which will help him choose the platform that suits him the most. A developer might decide to include some functionality in their new platform that lacks in the existing platforms and that functionality might make their platform successful.

\section{Literature Review}

A literature review was conducted on three categories of online recruiting platforms in order to reveal their main characteristics. A freelancing platform, a platform dedicated to students job finding and a general platform will be analyzed.

Nowadays, freelancing is one of the most desired ways of working. Working at one's own pace and being able to choose a preferred project attracted a considerable range of people looking for a suitable career. Freelancing also permits working remotely - the employee doesn't need to come daily to the company's premises. An example we consider relevant and one of the most popular for the scope of freelancing platforms is freelancer.com. In 2015 it won the award for the Best Employment Website in the 19th Annual Webby Awards and People's Voice. [1] The platform gives a wide range of skills for people looking for a job to choose from and an intuitive flow to follow from the creation of the account to the completion of the profile. [2]

In terms of the number of users, they claim to have more than 18 million users in 247 countries. It is ranked number 1496 in the world by Alexa, with most users coming from India, Pakistan and the United States. Also according to Alexa, the website loads very slowly, in about 3.8 seconds in average.[7]

The platform offers a vast amount of features. The design is responsive, so that the website looks great both on mobile devices and on PC. They have their own pages on social sites like Facebook and Twitter. Freelancer.com also comes as a mobile application for IOS and
Android. In terms of creating the account, initially the user is requested to choose between a number of categories which contain some skills each. No CV is requested. The user profile will then show a number of aspects which are considered to be representative for the person. Every user has a rating received by completing projects. Statistics are shown for every user, like the percentage of jobs successfully completed and how many of them were completed on time. A nice feature of the website is the ability to take exams and display the results on your profile page. The accounts can be verified with social sites, by phone or by email. The site can be used freely, but paid accounts also exist which offer some advantages like the ability to fill more skills on your profile or to place more bids. The search function is also based on the required skills to take the job, but other criteria can be checked like the money offered for the job, the location or the language. As a company, you can add your own projects. Among the required information, you can add a project description, the skills required for the project, your budget etc. Freelancer.com also tracks your money balance. Users can add friends and an instant messaging system is provided. Sending private messages is also possible.

Another important segment of the workforce around the world is represented by the students. A part time job is often preferred among the students for paying their university fees but also for acquiring experience for future full time jobs. For companies, hiring students is also preferable in a lot of scenarios. For example, for the tasks requiring a limited amount of work hours per day, a part time student employee is a good candidate. One platform that is focused on hiring students is stagiipebune.ro. Most of the jobs here are part time, available only to students from the most popular universities in Romania. The platform also provides an intuitive flow for a complete profile creation. [3]

The platform has over 1000 users, almost all of them students.[9] It is ranked number 1157278 by Alexa with almost all users coming from Romania. It loads slowly, in about 4 seconds.[10] 
The website doesn't offer a responsive design or mobile applications. It has its own pages on social sites. The user profile is $\mathrm{CV}$ based. The user needs to fill at least $50 \%$ of the CV before starting to apply for jobs. The CV contains information related to current studies, objectives after graduation, abilities, school or extracurricular activities and past jobs, if any. There isn't an actual search function. When searching for a job the user needs to select firstly the job domain and after that he'll be shown all the available positions, grouped by companies. The filtering ability resumes to the location of the job. Stagiipebune has its own blog and offers a contact form. Using the website is free for students. For companies, the free account is limited to 20 student applicants - for more, the use of paid accounts is mandatory. Unlike other recruitment platforms, stagiipebune also offers pages for universities.

There are also the classical online recruiting platforms. One example is hipo.ro, a platform designed for a wide range of people. Therefore, students, freelancers and other people specialised in different areas could apply here in the search for a matching company. [4] Hipo.ro has more than 320000 users and more than 400 employers. It is ranked number 93126 by Alexa with almost all users coming from Romania. The website seems to load fast, in about 1 second in average.[8]

In terms of features, Hipo.ro doesn't offer a responsive design. They have their own pages on social sites, but no mobile applications. The user profile is CV based. The user will create his own CV by filling his past experience, studies, abilities, a photo and information about the desired job. The search function lets the user do some advanced filtering, like the domain of the job, the kind of job (full or part time), the location or the required experience, but it won't automatically check his $\mathrm{CV}$ when doing a search - the user needs to fill the search details himself. Hipo offers a career page which displays useful news related to jobs and career improving. They also offer information about developer conferences which might be useful for IT people. Hipo.ro has collaboration with more than 130 compa- nies in their 'Top employers' section. The employer account is somehow harder to obtain as it is not created automatically, but requires the employer to send an email to Hipo.

In sum, there is a wide on the market of online recruiting platforms. There is important to note that the recruiting managers' challenges increase also as the market expands. Hence, there has to be set a balance between too verbose and too scarce information in the job description. Another important factor is the vividness of the description and the amount of interactivity between the recruiters and the job seekers. The recruiting managers have to consider the fact that a more thorough knowledge of the customer - the job seeker- will lead to a better job fit, but on the other side, a very detailed job profile available for the prospective employee to complete will lead to a more time-consuming procedure. [5]

On the other hand, a subjective feature such as the attractiveness of the recruitment websites is positively correlated with a good impression on the organization. [6] As a result, we will increase our focus on the interface section of our platform in order to create a positive user experience and a better impression on certain organizations.

\section{Methods}

Whether you are a recruiter or somebody that is looking for a job, you have to take into consideration a few aspects before choosing the online portal that suits you.

Usually, the grading scale of a website feature is composed of three levels, depending on the type of the characteristic that is analyzed. For volumes, the scale is composed of low, medium and high, for quality the three levels are bad, acceptable and good and for speed the levels are very slow, slow and fast. Other features are analyzed based on their presence or absence with a simple tick.

Because not all job boards are created equal, one important step in choosing the desired one is taking into consideration the quality and quantity of applicants each job board delivers, as well as the cost of these job boards. When grading the traffic volume of such a website, 
the method used was asking people which portals they have used.

Not knowing which job portal to use, determines the user to simply search Google for such a tool. Thus, it is inevitable to grade the quantity of inbound links present on different blogs, or online articles that Google returns as results. Also, most job portals use the concept of key words to help search engines find them based on user inputs and place the website in the top results. A simple way to determine which keywords are used, is to inspect the source of a page.

There are several features job portals should have. No matter the membership type, people are definitely interested if a $\mathrm{CV}$ is required or if there is a large range of industries or countries available or if there is a large range of skills users can choose from and if there is any feature that allows displaying special skills on a user's personal page. Most of the times, skills are the points that actually make a difference when a recruiter looks over several profiles. The more users they have, the bigger chance they have to show up in searches.

Another important aspect is the possibility to access the portal via social websites. The extent to which social networking has grown has created a very efficient entry for job portals. Users find it easier to sing up on a job portal using a social network where they already have an account. It doesn't matter if the account creation process is intuitive or not, this method is still preferred.

For most of the features named above the grading was done by simply browsing through the portal and searching for different aspects that other people thought to be very important.

Table 1. Comparative analysis

\begin{tabular}{|c|c|c|c|}
\hline & freelancer.com & stagiipebune.ro & hipo.ro \\
\hline Traffic volume & High & Low & High \\
\hline Quantity of inbound links & High & Low & High \\
\hline $\begin{array}{l}\text { Keywords strategy - easy to rank key- } \\
\text { words }\end{array}$ & $\mathbf{X}$ & $\checkmark$ & $\checkmark$ \\
\hline $\begin{array}{l}\text { SEO (visibility in search engine result } \\
\text { list)-non paid results }\end{array}$ & High & Medium & Medium \\
\hline Wide range of available industries & $\checkmark$ & $\bar{X}$ & $\checkmark$ \\
\hline High number of available countries & $\checkmark$ & $\mathbf{X}$ & $\mathbf{X}$ \\
\hline Site loading speed & Slow & Slow & $\begin{array}{l}\text { Very } \\
\text { fast }\end{array}$ \\
\hline Site description quality & Good & Good & Good \\
\hline $\begin{array}{l}\text { Organizers contact information dis- } \\
\text { played }\end{array}$ & $\sqrt{ }$ & $\sqrt{ }$ & $\sqrt{ }$ \\
\hline Wide range of skills to choose from & $\checkmark$ & $\mathbf{X}$ & $\sqrt{ }$ \\
\hline Intuitive account creation flow & $\checkmark$ & $\sqrt{ }$ & $\sqrt{ }$ \\
\hline Number of users & High & Medium & High \\
\hline Responsive design & $\checkmark$ & $\mathbf{X}$ & $\mathbf{X}$ \\
\hline Integration with social platforms & $\checkmark$ & $\sqrt{ }$ & $\sqrt{ }$ \\
\hline Compatibility with iOS and Android & $\checkmark$ & $\mathbf{X}$ & $\mathbf{X}$ \\
\hline User statistics(completed projects) & $\checkmark$ & $\mathbf{X}$ & $\mathbf{X}$ \\
\hline $\begin{array}{l}\text { Ability to test skills and display results } \\
\text { on personal page }\end{array}$ & $\checkmark$ & $\mathbf{X}$ & $\mathbf{X}$ \\
\hline Authentication via social sites & $\checkmark$ & $\mathbf{X}$ & $\checkmark$ \\
\hline Number of languages required & High & Low & Medium \\
\hline Average salary offered & High & Medium & High \\
\hline Number of jobs available & High & Medium & High \\
\hline
\end{tabular}




\begin{tabular}{|l|l|l|l|}
\hline $\begin{array}{l}\text { Social features (friends adding, instant } \\
\text { messaging) }\end{array}$ & $\checkmark$ & X & $\checkmark$ \\
\hline High number of available job types & $\checkmark$ & X & $\checkmark$ \\
\hline CV required & X & $\checkmark$ & $\checkmark$ \\
\hline Search filters & $\checkmark$ & X & $\checkmark$ \\
\hline Information about events & X & X & $\checkmark$ \\
\hline TOTAL & $\mathbf{4 4}$ points & $\mathbf{1 7}$ points & $\begin{array}{l}\mathbf{4 1} \\
\text { points }\end{array}$ \\
\hline
\end{tabular}

When searching for the best online job portal, the large number of users that access the page is as important as the number of times they do so. Also, the number of keywords a site contains is linked to the position in a search engine and to the number of inbound links that lead to this portal. Most users, candidates and employers, search for a large variety of features when it comes to job portals. These being said we can conclude that all the features mentioned in the above table are equally important.

Even though some features have the same grading system, each one of them has a different meaning. Obviously the high grade in the "traffic volume" feature is not the same as the high grade for the "SEO" feature. This is not the case for the features that are graded with checked or unchecked, though. This is simply a matter of the feature being present or not. For example when talking about a responsive design one of the aspects taken into consideration is the dynamic size of the application window on different devices.

For a better understanding we will explain the meaning of each grading system:

- Traffic volume:

- Low: <100 views per day

- Medium: 100-1000 views per day

- High: >1000 views per day

- Quantity of inbound links:

- Low: <10 links

- Medium: 10-20 links

- High: >20 links

- SEO (visibility in search engine result list)-non paid results:

- Low: 20-10 results returned by search engines

- Medium: 5-10 results returned by search engines
- High: first 5 results returned by search engines

- Site loading speed:

- Very slow: $>5$ seconds

- Slow: 5-2 seconds

- Fast: <2 seconds

- Site description quality:

- Bad: Imprecise and poor quality of details about the company, contact and services

- Acceptable: Precise but not enough details about the company, contact and services

- Good: Precise and detailed information about the company, contact and services

- Number of users:

- Low: <100.000 users in Romania / $<3$ million users worldwide

- Medium: 100.000 -200.000 users in Romania / 3 million-6 million users worldwide

- High: >300.000 users in Romania / > 6 million users worldwide

- Number of languages required:

- Low: 1 language

- Medium: 2 languages

- High: 3-4 languages

- Average salary offered:

- Low: <400 euros

- Medium: 400-600 euros

- High: >600 euros

- Number of jobs available:

- Low: < 100 jobs

- Medium: 100-300 jobs

- High: >300 jobs

Calculating the total score each website has, depends on the level of certain features or on its presence or absence. Thus the features that are marked with a tick have a score of two 
points and the ones marked with an $\mathrm{X}$ are worth no points. Same rule applies for the features that are graded using a scale: the lowest rank is worth no points (low, bad, very slow), the intermediate level is worth 1 point (medium, acceptable, slow) and the highest ones are worth 2 points (good, high, very fast). When a feature is not present or is graded with the lowest grade possible it is just natural to award it no points, no matter the grading scale chosen. Because between the lowest grade and the highest grade there is only one other, it has been decided to keep the same distance between the number of points available for awarding. Thus, the lowest grade is placed on the first position ( 0$)$, the intermediate one is on the second (1) and the highest one is placed third (2). To keep the same grading system it has been decided to grade the check with 2 points and the $\mathrm{X}$ with 0 points because there is no intermediate level.

After analyzing the data we can conclude the freelancer.com is the preferred solution and very close to it is hipo.ro. We have to take into account that freelancer.com is worldwide and hipo.ro is Romania oriented. These being said both sides are somewhat equal. The third one, stagiipebune.ro could be improved by adding a larger range of industries and job types.

\section{Results Analysis}

In order to analyze the characteristics shown in the table, we organized the results analysis into different sections.

\section{General web characteristics}

The analyzed web sites have different traffic volumes. This difference may be explained by the fact that freelancer.com is an international site, while the other two sites are Romanian. Thus, Hipo.ro has a good traffic volume Alexa ranking, for a local website. On the other hand, the users of stagiipenbune.ro are mostly college students in Information Technology, searching for a summer internship, therefore, the traffic volume is not uniformly distributed over the year and it is 10 times less than hipo.ro`s traffic volume.

The same logic will be applied to the quantity of inbound links. Freelancer.com appears on many blogs and tech websites at an international scale. Hipo.ro has strong partnerships with a broad range of Romanian companies, offering sponsorship to Top employers annual hiring event; it is therefore well referenced in news and social media.

All the three sites contain easy to rank keywords.

Regarding SEO (visibility in search engine result list)-non paid ads, Freelancer.com ranks first when searching for freelancing, while hipo.ro ranks only third on its category, after ejobs and bestjobs. Stagiipebune.ro ranks first when searching for "stagii".

Concerning the site loading speed, Freelancer.com loads slowly, but that can be explained by the location of the data center (we noticed that they use Amazon Web Services). Hipo.ro loads very fast (1 sec), being hosted in Romania. Having a python based backend, stagiipebune.ro loads slowly.

The site description quality is good for all the three analyzed sites.

\section{Portability}

Freelancer.com is the only site that scales on multiple screen sizes and also offers native Android and iOS applications.

\section{Miscellaneous HR specific features}

All the three sites offer intuitive account creation flow, and also do integrate with social platforms.

The number of users varies. In absolute terms, freelancer.com leads with 18 million users. Compared with geographical reach, the number of users for hipo.ro is large, but not the largest for the Romanian HR market. Stagiipebune.ro offers a modest user base, with only 1000 users, few of them being active during the whole year.

Related to the number of jobs available, Freelancer.com boasts with an impressive number of jobs posted - 8,722,425. Hipo.ro has a modest 3100 active jobs, but this number highly depends on the labor market's flow. Stagiipebune.ro has around 100 internships during Spring, when the internship season opens.

DOI: 10.12948/issn14531305/20.2.2016.02 
Hipo.ro has a high number of available job types in a variety of domains (industries), while Freelancer.com offers more jobs in Software Engineering. Frrelancer.com on the other hand has a high number of available countries, while on hipo.ro there are few jobs from abroad. Stagiipebune.ro focuses only on Information Technology jobs.

Freelancer.com has good search suggestions and search filters. Hipo.ro has decent search filters (Company Name, City), but sadly lacks search suggestions. The job cloud algorithm on hipo.ro is interesting, but fails to suggest good job criteria.

On all the three platforms, the employer's contact information as well as other job postings from the same employer are visible. Since the internship is just the beginning in the career, the ability to test skills and display results on personal page is only present on freelancer.com and on hipo.ro for some of the jobs posted.

$\mathrm{A} \mathrm{CV}$ is not required for freelancer.com, you have a profile with completed projects, while the other platforms do require a standard CV. Regarding the information about events characteristic, hipo.ro is also a laboring event organizer so it has news about upcoming events on their platform.

\section{Discussions}

There are substantial differences between the three platforms that we analyzed. Thus, it is very difficult to compare them and in some situations the results might be misleading. Depending on what you're looking for, all three platforms can be useful and might help you to find a job or, if you're a recruiter, to find the perfect candidate.

For example, the low traffic volumes of stagiipebune.ro don't matter so much if you're from Romania and you're a student. Students usually look for summer internships and from this point of view stagiipebune.ro is the most suitable.

Of course, on the other hand if you're not Romanian, hipo.ro and stagiipebune.ro are not valid options as they don't offer the possibility to change the website language. Moreover, stagiipebune.ro has partnerships with Romanian universities, so their internships are oriented to Romanian students.

If you're looking for international scale jobs, freelancer.com might be the most suitable platform as it has international cover and offers a wide number of jobs. On the downside, if you're not a software engineer freelancer.com might not be able to find you the job that you desire, as most of its available jobs are from this domain. In this case, a more general platform like hipo.ro is to be preferred.

SEO is a very important aspect, at least for the recruiter. Because a recruiter wants to maximize the number of competent users that will apply for the position, he might be tempted to post the job on a more visible platform in regards to SEO. On the other hand, a recruiter will post on stagiipebune.ro only if he has available internships; the same goes if he has available freelancing jobs: he will post on freelancer.

Based on these results, we can suggest some features that we believe would help the analyzed platforms to perform better.

Stagiipebune.ro could widen the range of available industries. It only has 2 industries at the moment: IT and economics, which limit the number of users that can use their platform. A wider range of industries would result in a greater number of users which might also improve their SEO, as more people will search them and visit their website. A better search system will make it easier to find a proper internship. Integration with mobile devices and social media is also very important nowadays and should be considered. They should improve the site loading speed.

Hipo.ro could increase their number of users by developing their own mobile application. A more detailed user profile page would be helpful for recruiters - for example, hipo.ro could allow users to take tests and display the results on their profile page. A very important step would be to become international - that will boost the number of users, available jobs and SEO. 
Freelancer should also allow users to post a $\mathrm{CV}$, as it is a standard, international recognised way of showing which skills a candidate has. They could also track freelancing events and suggest nearby events based on the users' skills. Also, they should improve the site loading speed.

The results concur with Maurer, S. and Liu, Y's opinion that there has to be a balance between too verbose and too scarce information in the job description [5] From this point of view, stagiipebune.ro offers too scarce information and as a result it ranked at the bottom in our study. Although freelancer.com offers a lot more complex information, if might be too verbose and thus it ranked almost equal with hipo.ro.

Attractiveness is a very important aspect, according to [6]. Freelancer.com has the most attractive design and we can consider that this aspect is what gives it the extra points in comparison with hipo.ro.

\section{Conclusions}

Most people don't know what online job portal suites them. They don't have knowledge about what a website has or doesn't have. It is very hard for both candidates and employers to put together such a document containing a detailed description of online job portals.

Pointing out the pros and the cons for each website, will not only help candidates and employers choose the most suitable platform but help the administrators and owners of these platforms to improve their workflow, to add requested features and to aid their users. Each one of us has been in the position of searching for a job. Sometimes we find the same company, with the same hiring opportunities on different portals. On some of them, the information may not be enough thus making us change our mind about applying to that job, when in fact the opportunity is attractive but is detailed on another website that has more features but is unknown to the public because it is missing features like keywords or SEO. The point of this analysis is to understand what users want and need and what is required to satisfy their hunger for data and infor- mation. It is important to understand that business clients prefer job portals to print media advertisements and why it is important to put effort in developing such applications.

We can conclude that the industry will perform well in the long run only if there are no economic pressures on it like that of depression, poverty etc. which ultimately lead to lack of jobs in the country/ world. But even if the portals keep on doing well, we should also keep in mind the relative competitions that they are already facing from other sectors like:

- Social Media like Linked in

- Campus Recruitments

- Internal Referrals

- Print Advertisements

People depend more on social sites for recruitment like that of LinkedIn and Facebook which are major threats to these job portals. Keeping all those factors in mind the Job portals should start creating their own values and differentiations in order to compete with their distant competitors instead of competing amongst others. More focus should be brought in to the rural market as it is a huge market which is still very much untouched and can be tapped. Great opportunities lie in the rural markets. The major advantage of the portals is that they provide numerous facilities apart from recruitment possibilities and this should be marketed properly.

Large players hold a big portion of the available market share, making it difficult for smaller players to compete within the same space. Major players Freelancer.com and Hipo.ro have well-established brand names, which help attract clients and users to their site. The level of competition among industry participants also serves as a barrier to potential entrants. New websites must have a specific market or demographic target before establishing a name for them. It is important to conduct further investigation to determine the impact that career fairs and the other aspects mentioned earlier (social media, campus recruitments, internal referrals etc.) have on online recruitment platforms. A comparison between social platforms and job portals must be made, pointing out the pros and cons for each of them. It is important to also find out 
the public opinion over this matter and to understand which solution people prefer.

\section{References}

[1] Webbyawards.com. (2016). [online] Available at:

http://webbyawards.com/winners/2015/websites/general-website/employment/freelancercom/ [Accessed 20 Mar. 2016].

[2] Freelancer.com. (2016). Freelancer - Hire \& Find Jobs. [online] Available at: http://freelancer.com [Accessed 20 Mar. 2016].

[3] Junio.ro. (2016). Voluntariat, part-time, internship și joburi ocazionale. [online] Available at:

http://junio.ro [Accessed 20 Mar. 2016].

[4] www.hipo.ro. (2016). Locuri de munca in

Romania pentru studenti, absolventi si tineri

profesionisti, Angajatori de top!. [online] Available at: http://hipo.ro [Accessed 20 Mar. 2016].
[5] S. Maurer and Y. Liu, (2007). Developing effective e-recruiting websites: Insights for managers

from marketers. Business Horizons, 50(4), pp.305-314.

[6] P. Braddy, A. Meade and C. Kroustalis, (2008). Online recruiting: The effects of organizational

familiarity, website usability, and website attractiveness on viewers' impressions of organizations.

Computers in Human Behavior, 24(6), pp.2992-3001.

[7] "Freelancer.Com Site Overview". Alexa.com. N.p., 2016. Web. 2 Apr. 2016.

[8] "Hipo.Ro Site Overview". Alexa.com. N.p., 2016. Web. 2 Apr. 2016.

[9] "Start Inscrieri La Stagii Pe Bune". Akcees. N.p., 2014. Web. 2 Apr. 2016.

[10] "Stagiipebune.ro Site Overview". Alexa.com. N.p., 2016. Web. 2 Apr. 2016.

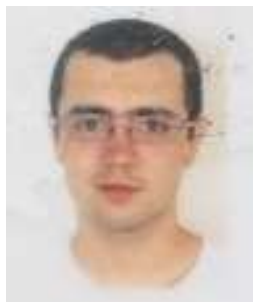

Gabriel CALIN has graduated the Faculty of Electronics, Telecommunications and Information Technology in 2012. Since then, he has been working as a Software Development Engineer in the billing department of Orange Romania. His work focuses on developing high quality applications, having an open mind to innovation and new technologies.

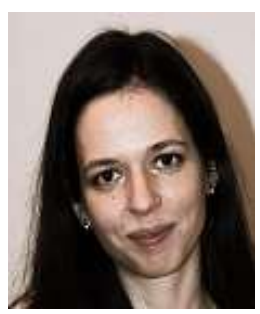

Iulia BIVOLARU has graduated the Faculty of Economic Cybernetics, Statistics and Economic Informatics in 2014. Since then, she has been working as a Software Developer at Cegeka. She is currently working in an Agile environment, developing a framework for banking applications. Her work is focused on creating software solutions which may improve people`s lifes.

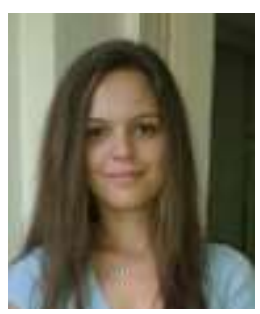

Paula IVAN has graduated the Faculty of Economic Cybernetics, Statistics and Informatics, Economic Informatics in 2014. Since then, she has been working as a full time software developer. She is focusing more on back-end code writing and Big Data analysis. Her current work focuses on creating connection bridges between Big Data services and other applications. 